\title{
ANALISIS DEL MARCO CONCEPTUAL DE LOS PRINCIPIOS DE CONTABILIDAD GENERALMENTE ACEPTADOS DE LOS ESTADOS UNIDOS DE NORTEAMERICA (NORMAS FASB)
}

\author{
ANALYSIS OF THE CONCEPT OF THE PRINCIPLES GENERALLY ACCEPTED \\ ACCOUNTING OF UNITED STATES OF AMERICA (FASB RULES)
}

\author{
Percy Vílchez Olivares ${ }^{1}$ \\ Docente Asociado de la Facultad de Ciencias Contables, UNMSM
}

(Recepción: Setiembre de 2009 / Conformidad: Octubre de 2009)

\section{RESUMEN}

El Marco Conceptual para la preparación y presentación de estados financieros, es un pronunciamiento que direcciona y fundamenta los principios de contabilidad generalmente aceptados de los Estados Unidos (US GAAP), y a su vez tiene vigencia en nuestro país, respecto a la aplicación de determinar los principios de contabilidad generalmente aceptados en el Perú, en su carácter supletorio de acuerdo a lo establecido por la Resolución No 013- 98 del Consejo Normativo de Contabilidad, órgano de la Contaduría Pública de la Nación. El Marco Conceptual es el eje central de un sistema normativo en el proceso de emisión y desarrollo de nuevas normas. Los consejos normativos de las norma internacional IASB y la norma americana FASB han iniciado desde el año 2006 un proyecto conjunto de mejorar y alinear sus Marcos Conceptuales, con la finalidad de lograr un conjunto de normas de alta calidad, basados que permitan impulsar la convergencia de la información financiera que se presenta y asimismo, contribuya al libre flujo de los capitales en los diferentes mercados. El presente artículo presenta un estudio analítico del Marco Conceptual de las normas emitidas por el Financial Accounting Standard Board FASB aplicable para las empresas peruanas que presentan información a su Matriz con sede principal en los Estados Unidos y reportan, a la Comisión de Valores de dicho país.

Palabras Claves: marco conceptual, convergencia de normas contables, objetivos y usuarios de la información contable, norma contable.

\begin{abstract}
The Framework for the preparation and presentation of financial statements is a statement that directs and informs the accounting principles generally accepted in the United States (U.S. GAAP), which in turn is valid in our country, on the application to determine the accounting principles generally accepted in Peru, in its supplementary nature as established by Resolution No. 013-98 Accounting Standards Council, an organ of the Public Accounts of the Nation. The Framework is the backbone of a regulatory system in the process of issuing and development of new standards. The policy advice of international standard American Standard IASB and FASB have initiated since 2006 a joint project to improve and align their framework with the aim of achieving a set of high quality standards, enabling push-based convergence financial information is presented and also contribute to the free flow of capital in various markets. This article presents an analytical study on the framework of the rules issued by the Financial Accounting Standards Board FASB applies for Peruvian companies that report to its parent company headquartered in the United States and report to the Securities and Exchange Commission that country .
\end{abstract}

Key words: conceptual framework, convergence of accounting standards, objectives and users of accounting information, accounting standard.

Contador Público Colegiado Certificado, UNMSM. Director Académico de la Facultad de Ciencias Contables, UNMSM. Magister en Ciencias Contables y Empresariales. E-mail: pvilchez20@hotmail.com 


\section{INTRODUCCIÓN}

Partiendo de una perspectiva de negocios, la contabilidad en su proceso de emisor de información financiera busca satisfacer necesidades de información de los usuarios, y en su caso de los mercados de capitales.

Los Inversionistas y los Analistas de Inversión, en el proceso de canalización y evaluación de inversiones financieras analizan información financiera de empresas de países diferentes preparados de acuerdo a criterios diferentes que limitan el análisis decisorio de la inversión. Asimismo, las diferentes normas contables ocasionan que la información financiera que presenta una misma empresa varía de forma importante según se apliquen una $\mathrm{u}$ otras normas, confundiendo al inversionista.

En un mundo globalizado, tiene más sentido que una transacción económica sea contabilizada de forma similar, con independencia de donde se realice. De esta forma se incentivaría la Inversión Internacional a mayor escala y los Inversionistas y Analistas de Inversiones podrán contar con información financiera más homogénea y eliminar los elevados costos para la preparación de estados financieros.

Por lo descrito anteriormente, en la profesión contable se ha generado un interés en la convergencia de normas contables entre normas IASB y normas FASB, que busca uniformizar las prácticas contables a nivel internacional y en los estados unidos de Norteamérica.

El presente artículo de investigación se orienta a analizar el contenido del Marco Conceptual del FASB como base inicial para poder comprender la factibilidad de lograr la convergencia de ambos modelos normativos, y que impacto ha tenido en nuestro país la aplicación supletoria de las normas FASB.

\section{ANÁLISIS DEL MARCO CONCEPTUAL DE LAS NORMAS FASB}

\subsection{Antecedentes y Pronunciamientos Técnicos}

Los Antecedentes a las Normas Nacionales de los Estados Unidos se centran a la Asociación Americana de Contadores Públicos (AAPA, por sus siglas en inglés), uno de los organismos más importantes en pronunciamientos autorizados en principios contables; donde por el año 1917 fue reorganizado, pasando a denominarse Instituto Americano de Contadores (AIA, por sus siglas en inglés), y tomando el nombre actual en 1957 de Instituto Americano de Contadores Públicos Certificados (AICPA, por sus siglas en inglés). Al respecto, el AIA en 1920 crea el Comité de Terminología, emitiendo 11 Boletines de Terminología Contable llamados ATB, con la finalidad de recopilar y clasificar los distintos conceptos contables utilizados hasta ese momento. Este Comité tuvo vigencia hasta 1929. Con la profunda crisis económica de 1929 producto del crack bursátil de Nueva York, los Estados Unidos prestan gran importancia al tema contable. $\mathrm{Al}$ respecto, entre 1930 y 1933 el AIA y la Bolsa de Valores de Nueva York crean un comité cuyo objetivo era aconsejar a la Bolsa en temas contables. Este Comité en 1938 junto con los otros del AIA se unificó en un Comité denominado Comité de Procedimientos Contables (CAP, por sus siglas en inglés) para abordar temas contables específicos. El Comité de Procedimientos Contables, desde 1938 a 1959 emitió 51 Boletines, de Investigación Contable (ARB, por sus siglas en inglés).

Otro aspecto relevante en la historia de la Normatividad Contable Americana; es que en 1959 el Comité de Terminología del AICPA y el Comité de Procedimientos Contables (CAP) fueron sustituidos por la Junta de Principios Contables (APB, por sus siglas en inglés) del AICPA con el fin de resolver temas contables específicos, $y$ desarrollar una estructura teórica contable con un marco amplio de principios contables coordinados y estructurados sobre la base de postulados contables.

Las declaraciones del APB se denominan APB opiniones, La Junta de Principios Contables, desde 1959 hasta 1972 emitió 31 Opiniones de Principios Contables (APB Opinions, por sus siglas en inglés). Pero el APB durante la década del sesenta fue duramente criticado por el sector empresarial y de gobierno, principalmente por contar con miembros que participaban de la emisión de normas contables y mantienen vinculación laboral con el sector empresarial, generando conflicto de 
interés y adicionalmente, por la conceptualización de su normativa contable.

En ese sentido, que el AICPA en 1971 creó un grupo de estudio para revisar la polémica establecida en la conceptualización de su normativa (positivismo vs normativismo) y para evaluar el conflicto de intereses, los estudios de éste grupo presidido por Francisco Wheat concluyeron en 1972, recomendado la creación de la Junta de Normas de Contabilidad Financiera (Financial Accounting Standards Board, FASB, por sus siglas en inglés).

En 1973 y con la finalidad de suplir la falta de eficiencia del APB, en lo relativo al Marco Conceptual $^{1}$, este organismo es reemplazado por el FASB cuya primera meta fue formular un sistema coherente e interrelacionado de objetivos y bases fundamentales que pudieran conducir a la emisión de normas consistentes, que indicaran la naturaleza, función y límites de la información financiera.

El FASB, es un Organismo Profesional Contable de los Estados Unidos, con ámbito privado encargado de elaborar normas contables, compuesto por miembros que están remunerados y que trabajan a dedicación exclusiva. El FASB es un Organismo independiente. No es parte del AICPA como fue su antecesor.

El FASB es el órgano autorizado por la profesión Contable para emitir Principios de Contabilidad Generalmente Aceptados en los Estados Unidos (US GAAP) según consta en el Rule 203 del Código Ética del AICPA y de acuerdo a las directrices de la US.SEC (securities and Exchange Commission en inglés), agencia de gobierno de Estados Unidos que reafirma la política de dependencia de la SEC al sector privado para la emisión de Normas Contables.

Los US GAAP son los Principios de Contabilidad Generalmente Aceptados de los Estados Unidos de América. En la actualidad, estos principios son emitidos por la Junta de Normas de Contabilidad Financiera (FASB).
El marco normativo del FASB, a la fecha está constituido por un Marco Conceptual expuesto en FASB conceptos, los pronunciamientos de FASB conocidos por el nombre de Declaraciones sobre Normas de Contabilidad Financiera y las Interpretaciones de FASB. A la fecha se posee el siguiente cuerpo normativo contable:

- Declaraciones sobre Conceptos de Contabilidad Financiera (SFAC, por sus siglas en inglés).

- 163 Declaraciones sobre Normas de Contabilidad Financiera de FASB (SFAS, por sus siglas en inglés).

- 48 Interpretaciones de FASB (FIN, por sus silgas en inglés).

- Diversos APB y ARB que a la fecha siguen vigentes.

\subsection{Declaraciones del Marco Conceptual FASB}

El FASB después de doce años de trabajo, en 1985 emitió sus «Declaraciones de Conceptos sobre Contabilidad Financiera «cuya finalidad es pretender poner de manifiesto los objetivos y fundamentos que constituyen la base para el desarrollo de las normas de contabilidad financiera y presentación. Estas Declaraciones de Conceptos constituyen el Marco Conceptual del FASB.

Las Declaraciones se pueden estructurar en diversos conceptos que se abordan pero que se pueden estructurar en los siguientes: usuarios y objetivos de los estados financieros, las hipótesis contables básicas, las características cualitativas de la información contable, elementos de los estados financieros, y reconocimiento y medición de los elementos de los estados financieros.

El FASB define su Marco Conceptual ${ }^{2}$ como un entramadológico deductivo donde seinterrelacionan objetivos y conceptos, proporcionando coherencia y credibilidad al proceso de normalización e información externa útil para la toma de decisiones al usuario externo. 


\subsubsection{Usuarios y Objetivos de la información financiera}

El SFAC 1 señala que los principales usuarios de la información financiera son aquellos que basan sus decisiones en sus relaciones con las entidades y en el conocimiento que poseen acerca de ellas.

La lista de los principales usuarios de la información indicados son: propietarios, acreedores, proveedores, inversionistas potenciales, clientes, empleados, administradores, directores, analistas financieros, abogados, economistas, autoridades tributarias, organismos controladores, legisladores, asociaciones de comercio, profesores y estudiantes, y público en general.

Los objetivos de la información contable ${ }^{3}$ precisados en las declaraciones del FASB son las siguientes:

a) La información financiera debe suministrar información útil para ayudar a actuales o potenciales inversionistas, acreedores y otros usuarios, para que puedan tomar decisiones racionales de inversión, créditos y otras similares y la información debe ser comprensible, para aquellos que tienen una comprensión razonable de los negocios y actividades económicas y se encuentren dispuestos a estudiar y evaluar la información con razonable diligencia.

b) La información financiera permitirá un conocimiento de la situación financiera de la empresa y servirá para ayudar a los inversionistas, acreedores y otros usuarios a evaluar su liquidez y solvencia; un conocimiento del desempeño económico durante un período a través de la información relativa de rendimiento y resultados de la empresa.

c) La información financiera debe brindar información que ayude a los inversionistas y acreedores actuales y potenciales y otros usuarios en la evaluación de los montos, oportunidad e incertidumbre de los ingresos de fondos prospectivos provenientes de aportes, préstamos, dividendos, intereses o recursos autogenerados, y la correcta utilización de los flujos de efectivo de manera de no afectar su liquidez y solvencia.

$\mathrm{Al}$ respecto, el término «información financiera» es más amplio que el término de estados financieros, pero se debe definir y delimitar que es y que no es información financiera, pues por ejemplo un presupuesto de caja es usualmente circunscrito al concepto de información financiera, pero no es una información sujeta al campo normativo del FASB.

Asimismo, el FASB tiene una marcada orientación a atender la demanda de información del usuario en materia de flujos de efectivo; tales como, recursos disponibles, el efecto de las transacciones en efectivo y el nivel de financiamiento generado. En ese sentido, se observa una clara evolución den el concepto de información financiera relevante hacia los flujos de efectivo en detrimento del patrimonio.

\subsubsection{Hipótesis Contables Básicas}

Las hipótesis contables básicas están presentes en todo el modelo contable del sistema normativo definido. Aunque generalmente se definen como principios, epistemológicamente no corresponden a esa categoría, por el contrario son supuestos o proposiciones fundamentales, concernientes al medio económico y social en que se desarrolla la contabilidad.

De acuerdo al Marco Conceptual del FASB, las hipótesis contables orientan las bases del cual se puede desarrollar el modelo contable, y sobre las que descansan las características cualitativas de la información contable, los elementos de los estados financieros y los criterios de reconocimiento y medición de los elementos de los estados financieros. Las seis hipótesis básicas son: Entidad Económica, Empresa en Marcha, Unidad Monetaria Estable, Mercado de Libre

\footnotetext{
${ }^{3}$ SFAC 1 emitido en 1978
}

${ }^{4}$ SFAC 2 emitido en 1980 
Competencia, Periodicidad, y Base de Devengado.

La hipótesis de entidad económica, se refiere a que la empresa debe ser considerada como una entidad económica separada y distinta de sus propietarios y de cualquier otra unidad de negocios. Empresa en marcha, se refiere a que la entidad se considera como una empresa en marcha, es decir continuará sus actividades operativas en el futuro previsible. Unidad monetaria estable, se refiere a que las transacciones y hechos contables deben ser medidos utilizando un denominador común y unidades monetarias estables; excepcionalmente se puede utilizar la unidad monetaria de poder adquisitivo constante.

La hipótesis de mercado de libre competencia, se refiere a que las transacciones que se han realizado en un mercado de libre competencia, se realizan entre dos partes independientes y capaces de defender sus propios intereses. Periodicidad, se refiere a que el período mínimo normal para entregar esta información debía ser un año. Claro esta, que las empresas pueden preparar información en períodos de tiempo más cortos de acuerdo a sus necesidades de información. Y base de devengado, significa que los efectos de las transacciones y demás hechos contables se reconocen cuando ocurren independientemente al flujo financiero del cobro o pago por la transacción.

Respecto a las hipótesis planteadas por el FASB, debemos precisar que las hipótesis que se deben plantear en el Marco Conceptual, deben ser los supuestos fundamentales en que opera el modelo contable y deben provenir de bases eminentemente de la práctica contable y no de supuestos del mundo de los negocios y que de alguna manera le pertenecen también a otros campos del conocimiento tales como la administración, la economía, etc. Al respecto las hipótesis entidad económica, unidad monetaria estable, mercado de libre competencia, y período, constituyen conceptos contables del mundo de los negocios donde se desarrolla la norma contable, y no son parte de la normativa, por el contrario son parte de la teoría contable.

Debemos precisar que el modelo normativo del IASB considera como hipótesis contables a: empresa en marcha y base de devengado donde las otras hipótesis contables incluidas por el FASB, no son mencionadas, entendiéndose que se refieren a conceptos vinculados al mundo de los negocios y no propiamente a hipótesis contables.

\subsubsection{Características Cualitativas de la Información Contable}

El SFAC 2 establece las características cualitativas de la información contable ${ }^{4}$ precisando además el orden de jerarquía entre ellas. A partir de la definición de que información fuera útil para un propósito determinado del usuario; se definen las características cualitativas que hagan útil dicha información. De esto se deriva que pueden existir diferentes tratamientos contables posibles para el reconocimiento de un determinado hecho contable, ante esto de debe elegir aquella que sujeta a las consideraciones sobre su costo, entregue la información más útil para la toma de decisiones por parte de los usuarios de la información contable.

En esta declaración se precisa como once características cualitativas de la información contable, siendo la relevancia y la fiabilidad de la información, los ejes principales en que se transforman la base del resto de las cualidades de la información contable.

Según el FASB, la información contable debe poseer simultáneamente cuatro características primarias y secundarias. Las características primarias son la relevancia y la confiabilidad; y las características secundarias son la comparabilidad y consistencia. Estas características se basan a su vez en otras cualidades y en algunas restricciones. A continuación, se presenta el Cuadro $\mathrm{N}^{0} 1$ que muestra la jerarquía de las características cualitativas de la información contable, de acuerdo a lo señalado por el SFAC 2.

\footnotetext{
${ }^{4}$ SFAC 2 emitido en 1980
} 


\section{Esquema Jerárquico de las Características de la Información Contable}

\author{
Usuarios de la \\ Información \\ Contable \\ Restricción General \\ Características \\ Específicas para \\ el usuario \\ Características \\ Primarias \\ Ingredientes de las \\ Características Primarias
}

Características

Secundarias

Segunda restricción

para el reconoci miento

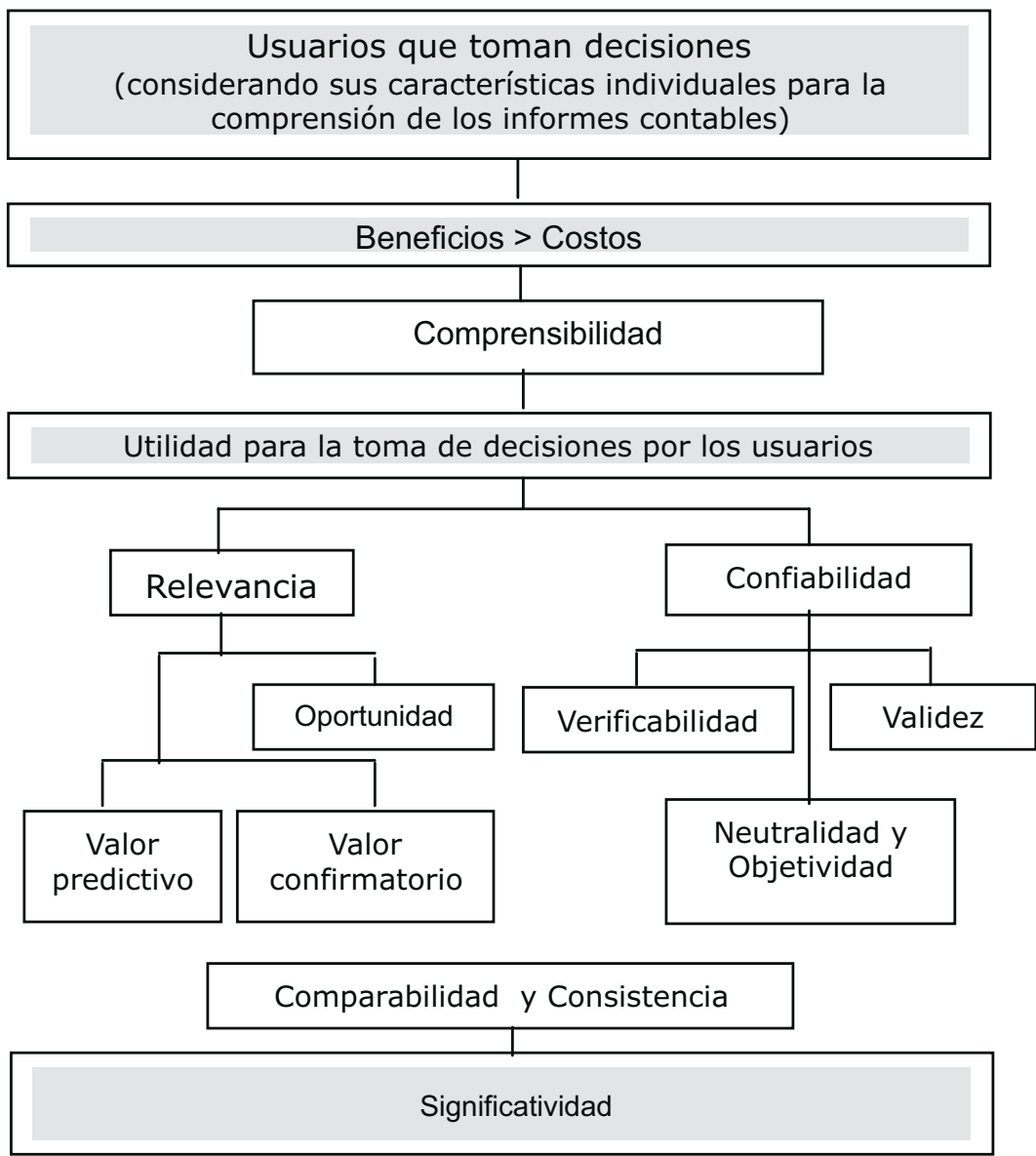

Considerando que la información contable debe ser útil en el proceso de toma de decisiones, por ello se precisa como característica especifica para los usuarios la Comprensibilidad, en tal sentido, la información contable debe ser fácilmente comprensible por usuarios con conocimiento razonable de los negocios y actividades económicas, y con voluntad de estudiar con razonable diligencia. No obstante ello la información sobre temas complejos debe ser incluida por razones de su relevancia, a pesar que sea difícil su comprensión para ciertos usuarios. La característica de Comprensibilidad sirve de enlace entre las diversas características de los usuarios de los informes contables y las características específicas de la información contable.

Las características primarias de la información contable son Relevancia y Confiabilidad. A fin de que la información sea relevante, ella tiene que poseer la capacidad de generar una diferencia para usuarios de la información contable. Se considera que una información es relevante, cuando su conocimiento impulsa a tomar o a variar una decisión. Para ello, la cualidad de relevancia, tiene su sustento en tres requisitos o cualidades: que la información tenga valor predictivo, valor confirmatorio y que sea oportuna. Asimismo, para que la información contable sea confiable, debe estar razonablemente libre de errores o prejuicios y representar fehacientemente lo que persigue representar. Para ello, la cualidad de confiabilidad requiere el cumplimiento de tres requisitos o cualidades: que la información sea verificable, sea neutral y que represente una imagen fiel.

Por otra parte, las características secundarias de la información contable son Comparabilidad y 
Consistencia. La característica de comparabilidad permite a los usuarios identificar las similitudes y diferencias entre dos grupos de hechos económicos debido a que tales similitudes y diferencias no fueron distorsionadas por el uso de políticas contables diferentes.

La comparabilidad tiene como: la uniformidad, que significa que debe seguirse la aplicación uniforme de políticas contables en la preparación y presentación de información contable. Ello no significa que las empresas no deban modificar políticas contables, en tanto existan otras más relevantes y confiables. Asimismo, La característica de consistencia se refiere al uso de los mismos procedimientos contables, ya sea de período a período dentro de una entidad o en un solo período en todas las entidades.

Las características de la información contable que han sido comentadas deben ser consideradas a la luz de dos restricciones: la Relación Costo Beneficio que implica revelar información que brinde beneficios a los usuarios que superen los costos de prepararla y la Significatividad que implica revelar aquella información que sea significativa.

$\mathrm{Al}$ respecto, las características de la información contable coinciden con las planteadas por el marco conceptual IASB, pero la diferenciación esta en que el marco conceptual FASB precisa nivel primario y se secundario para las características de la información contable.

\subsubsection{Elementos de los Estados Financieros}

El SFAC 6 establece los elementos de los estados financieros ${ }^{5}$, que agrupan por categorías las diversas características de los recursos económicos que se encuentran presenten o fluyen hacia la entidad.

En el caso del balance general los elementos que miden la situación financiera son el activo, pasivo, patrimonio, aportes de capital, retiros de capital, resultado global. En el estado de ganancias y pérdidas, los elementos son los ingresos, los gastos, ganancias y pérdidas.
El activo, son recursos propios o controlados por la empresa como consecuencia de transacciones pasadas, que probablemente generarán beneficios económicos futuros. El pasivo, corresponde a obligaciones presentes de la entidad a favor de terceros, que probablemente significarán un sacrificio económico; pero que persiguen un beneficio futuro. El patrimonio, es el monto residual de los activos de una entidad, después de deducir las obligaciones.

Los aportes de capital, son los aumentos en el patrimonio de una entidad que provienen de los aportes que efectúan los accionistas o socios. Otra forma de aumento de capital es la capitalización de utilidades o reservas, entre otras. Los retiros de capital, son las disminuciones en el patrimonio como consecuencia de la transferencia de activos o la prestación de servicios o pasivos asumidos por la institución a favor de los accionistas o propietarios. El resultado global, es el cambio en el patrimonio de la empresa como consecuencia de transacciones realizadas por ésta, excluyendo los aportes o retiros de los accionistas o propietarios.

Los ingresos, son las entradas de dinero u otros aumentos del activo o disminución del pasivo, como consecuencia de actividades propias del giro de la entidad. Los gastos, son las salidas de efectivo u otras disminuciones de activo, o aumentos de pasivos, necesarios para el desarrollo de la empresa y que ayudan a generar los ingresos. Las ganancias, son los incrementos en el patrimonio derivados de hechos extraordinarios, ajenos al giro de la entidad. Las pérdidas, son las disminuciones en el patrimonio de la entidad derivadas de hechos extraordinarios, ajenos al giro de la entidad.

El Marco Conceptual no identifica ningún elemento exclusivo del estado de cambios en el patrimonio neto ni del estado de flujos de efectivo, pero ambos si combinan elementos del balance general y del estado de ganancias y pérdidas.

SFAC 6 emitido en 1985 


\subsubsection{Reconocimiento y Medición de los Elementos de los Estados Financieros}

El SFAC 5 establece los criterios de reconocimiento de los estados financieros ${ }^{6}$, el criterio de reconocimiento es el proceso de registro de una partida en los estados financieros de una entidad como un activo, pasivo, patrimonio, ingresos, gastos, ganancias y pérdidas. Debe reconocerse toda partida que cumpla la definición de elemento siempre que:

- La partida debe identificarse con un elemento de los estados financieros.

- La partida tenga un costo o valor que pueda ser medido en términos monetarios.

- La partida tenga valor informativo, es decir relevancia para los usuarios de la información contable.

- La información debe ser registrada y presentada con confiabilidad.

Estos criterios de reconocimiento ${ }^{7}$, deben ser cumplidos en su totalidad y en el mismo orden en que han sido enunciados. Respecto a los criterios de medición de los elementos de los estados financieros, el SFAC 5 señala que las bases de medición o determinación de los importes monetarios en los que se reconocen los elementos de los estados financieros son: costo histórico, costo de reposición, valor de mercado, valor neto de realización y valor presente de los flujos de caja.

El costo histórico, es el precio pagado o adeudado por activos a la fecha de adquisición, los pasivos incluyen obligaciones que son medidos según los beneficios históricos recibidos cuando la obligación fue contraída. El costo de reposición, es el precio que se pagaría para reponer un activo o uno similar. El valor de mercado, es el precio que se podría obtener al vender un activo en una liquidación ordinaria. El valor neto de realización, es el monto que se espera recibir o pagar en la conversión de activos o pasivos, mediante sus costos directos de realización. El valor presente, es el monto de los futuros flujos de caja descontados a su valor presente.

$\mathrm{Al}$ respecto, el marco conceptual de las normas FASB a diferencia del marco conceptual de las IASB, consideran el costo de reposición como criterio de medición de los elementos de los estados financieros esta diferencia es sustancial y constituye el principal obstáculo a superar en el proceso de convergencia de ambos marcos conceptuales. Con relación a este punto, el FASB no indica un criterio de medición preferible a todos los activos y pasivos, esta Declaración sugiere la utilización de diferentes criterios, y la Junta del FASB debe seleccionar el criterio adecuado en cada caso particular, considerando el que entregue una información más útil y a un precio razonable.

Debemos precisar que el modelo contable estadounidense se basa fundamentalmente en el criterio de medición a costos históricos, debido a ser considerado como el más objetivo y confiable y en casos específicos se utilizan los otros criterios de medición. La preferencia por el costo histórico $^{8}$, tiene su fundamento en la falta de tratamiento de la incertidumbre, potenciando de esta manera, la fiabilidad de la información. El FASB al establecer como criterio de medición preferente el costo histórico y la hipótesis contable base del devengado no resulta operativo para proporcionar información útil sobre los flujos de caja futuros y su evolución, pero estos criterios e hipótesis permiten obtener información básica para su preparación.

II. LAS NORMAS FASB Y SU APLICACIÓN EN EL SISTEMA NORMATIVO CONTABLE EN EL PERÚ

\subsection{Análisis de la Regulación Contable en el Perú}

Con la vigencia Ley General de Sociedades Ley No 26887 de 1997, se estableció que los estados

\footnotetext{
6 SFAC 5 emitido en 1984

María García Castañeda (2007, pág. 89)

${ }^{8}$ Ana José Bellostas (1982, pág. 83)
} 
financieros se preparan y presentan aplicando las disposiciones legales y con Principios de Contabilidad Generalmente Aceptados.

Al respecto, El Consejo Normativo de Contabilidad, organismo de la Contaduría Pública de la Nación, emitió la Resolución $\mathrm{N}^{\mathrm{o}}$ 013-98 EF/93-01 de 1998, en la que señala que los Principios de Contabilidad Generalmente Aceptados son las Normas Internacionales de Contabilidad oficializadas en el Perú por el Consejo Normativo de Contabilidad, órgano de la Contaduría Publica de la Nación.

Dicha Resolución señala que por excepción y en aquellas circunstancias que determinados procedimientos operativos contables no están normados por el Comité de Normas Internacionales de Contabilidad (IASC) supletoriamente, se podrá emplear los principios de contabilidad aplicados en los Estados Unidos de Norteamérica (US GAAP).

Lo anterior, no excluye a las empresas peruanas que tienen inversión americana y reporten a su matriz de acuerdo a normas FASB y también aplicable a aquellas empresas peruanas que tienen sus acciones registradas y cotizadas en las bolsas de valores de los Estados Unidos, para el cual deberán reportan de acuerdo a principios de contabilidad generalmente aceptados en los Estados Unidos

\subsection{Análisis de Entidades Peruanas que Reportan a entidades de los Estados unidos}

Las empresas Peruanas que cotizan en Bolsa de Valores de los Estados Unidos o tiene su matriz en dicho país deben reportar estados financieros de acuerdo a normas IASB con una conciliación entre los resultados obtenidos de acuerdo a normas IASB y las normas FASB.

Se ha identificado a 7 empresas Peruanas (Principales o Subsidiarias) que cotizan en la bolsa de New York y preparan sus Estados financieros de acuerdo a regulación americana, el análisis de esta información ha tenido significativas dificultades en el acceso de información por el cual se ha realizado el análisis a 2 empresas 9 . Del análisis efectuado a algunas entidades se encontró las siguientes diferencias:

a) Reconocimiento de gastos de desarrollo. De acuerdo con las normas FASB, los costos de desarrollo se reconocen como gastos cuando se incurre. Esta normativa presenta diferencias con las norma IASB $N^{\circ} 38$ en la que es posible reconocer como activo, cuando cumple determinados criterios. Lo anterior tuvo un impacto significativo en la empresa minera Yanacocha, por ejemplo en los estados financieros al 31 de diciembre del 2005 de acuerdo a NIIF se obtuvo utilidad de \$ 535,476 miles de dólares y de acuerdo a normas US GAAP se obtuvo una utilidad de 525,474 miles de dólares.

b) Las combinaciones comerciales. De acuerdo con las normas FASB el impacto en los resultados principalmente relacionado a las combinaciones comerciales y el efecto de presentación de interés minoritario es más significativo que respecto a las normas IASB. Lo anterior tuvo un impacto significativo en la empresa telefónica, por ejemplo en los estados financieros consolidados al 31 de diciembre del 2005 de acuerdo a NIIF se obtuvo utilidad 4,827, millón de dólares y de acuerdo a normas US GAAP se obtuvo una utilidad de 4,144 millón de dólares.

\section{CONVERGENCIA DE MARCOS CONCEPTUALES}

\subsection{Antecedentes del Proceso de Convergencia}

Estados Unidos, ha iniciado un proceso de convergencia entre la norma contable FASB y las normas IASB. El evento más importante ocurrido a favor de la convergencia de las normas contables a nivel mundial, es el ocurrido en Setiembre del 2002 en Norwalk (USA) con la vinculación formal entre el IASB y el FASB, celebrando el «Acuerdo de Norwalk», donde ambos acuerdan seguir un Programa de Convergencia orientado a eliminar las diferencias existentes entre los dos modelos

\footnotetext{
${ }^{9}$ Capcha Jesús y otros. Trabajo Interamericano presentado en la Conferencia Interamericana de Contabilidad. Bolivia. (2007, Pág. 22-23).
} 
de normas contables. Para lograr dicho objetivo, los dos organismos contables establecen dos compromisos básicos:

a) Desarrollar normas de alta calidad que sean compatibles entre sí, mediante la eliminación de una serie de diferencias entre las Normas Internacionales de Información Financiera y los Principios de Contabilidad Generalmente Aceptados, en los Estados Unidos que no requieren de amplia reconsideración y son de resolución rápida.

b) Eliminar una serie de diferencias sustantivas entre las Normas Internacionales de Información Financiera y los Principios de Contabilidad Generalmente Aceptados, a través de la identificación de soluciones comunes. Para el logro de éste compromiso se ha preparado un documento denominado «Proyecto Específico de Convergencias». Siendo uno de los proyectos específicos de convergencia el de la convergencia de los Marcos Conceptuales.

Asimismo, ambas organizaciones profesionales han fijado agenda común en Proyectos en los cuales trabajar en forma coordinada y adicionalmente, existe la presencia por parte de un miembro del IASB en las oficinas de FASB para facilitar el intercambio de información y la cooperación entre ambas organizaciones. Por otro lado, los proyectos de IASB son monitoreados por el FASB a través de un miembro del FASB asignado exclusivamente a ésta tarea.

Para dimensionar la importancia de la convergencia entre estos modelos normativos, podemos señalar que unas 13,000 empresas tienen registrados sus valores en la Comisión Norteamericana del Mercado de Valores (SEC), donde un $10 \%$ aproximadamente de estas empresas son extranjeras. Si estas empresas extranjeras presentan estados financieros según NIIF o PCGA locales en lugar de US GAAP, es obligatoria una conciliación de los beneficios y el patrimonio neto con las cifras según USGAAP.

\subsection{Convergencia de Marcos Conceptuales}

El Consejo Normativo del IASB y FASB en el año 2006 emitieron un proyecto conjunto para mejorar y alinear sus Marcos Conceptuales, documento que a la fecha sigue siendo revisado. Este debate sobre el Marco Conceptual es esencial, porque ayudara a modelar las normas contables en los años venideros ${ }^{6}$. Aunque creemos que se necesita una revisión de los fundamentos del modelo de información, coincidimos en que los consejos deben, como parte de su proyecto actual, revisar urgentemente las áreas en que las normas presentes no son consistentes entre ellas o con los marcos conceptuales.

De lograr una convergencia de los Marcos Conceptuales se constituirá en el documento principal para ambos entes normativos, y se constituye en un paso deseable y necesario para la convergencia de las normas que emiten ambos organismos, es decir la convergencia de las Normas Internacionales de Información Financiera (NIIF) y los Principios de Contabilidad Generalmente Aceptados de los Estados Unidos (US GAAP).

En conclusión, la visión de crear un lenguaje mundial común en la contabilidad, se empieza a materializar con las iniciativas tomadas por dos organismos Líderes de la Profesión Contable. Sin embargo, considerando la complejidad de las diferencias por resolver estimamos que muchas de ellas se mantendrán hasta después del año 2012.

\section{CONCLUSIONES}

1. La Convergencia de los Marcos Conceptuales de las normas IASB y las normas FASB es un paso importante en la viabilidad la convergencia de las normas contables de ambos emisores de normas. El proceso de convergencia entre normas IASB

${ }^{10}$ Price Waterhouse Coopers. Carta Recordatoria (2007, pág. 99) 
y normas FASB se encuentra en la última etapa; sin embargo considerando las diferencias aun existentes no creemos que antes del 2012 se culmine dicho proceso.

2. La revisión de ambos modelos conceptuales permitirá definir prioridad y orientación de información respecto a los usuarios de la información contable emitida bajo el nuevo modelo normativo; asimismo, conciliar las diferencias existentes en las hipótesis contables de ambos modelos normativos

3. El FASB tiene una marcada orientación a atender la demanda de información del usuario en materia de flujos de efectivo. En ese sentido, se observa una clara evolución del concepto de información financiera relevante hacia los flujos de efectivo en detrimento del patrimonio.

4. Las empresas peruanas al preparar información contable de acuerdo a las normas IASB en el proceso de reportar los informes contables de subsidiarias a entidades matriz ubicadas en Estados Unidos o empresas peruanas que cotizan en bolsa de valores de Estados Unidos, realizan un proceso adicional y costos en la elaboración de la reconciliación de resultados obtenidos de acuerdo a normas IASB y las normas FASB.

\section{REFERENCIAS BIBLIOGRÁFICAS}

1. Bellostas A. (1992). Análisis de la consistencia en la estructura del marco conceptual. Revista Española de Financiación y Contabilidad, volumen XXII No 70. España.

2. Capcha J.,Salazar A. y Vilchez P. (2007) . Trabajo presentado en la Conferencia Interamericana de Contabilidad: Estudio de las Normas contables desde una perspectiva Lógica y Epistemológica y su utilización en los países de América. Bolivia,

3. Financial Accounting Standards Board (FASB) (1985): Statement of Financial Accounting Concepts No 1,2,5 y 6. Estados Unidos.
4. García María. (2003). «Fundamento y Normativa de la Contabilidad». Segunda edición., Editorial Lexis Nexis, Chile,

5. Price Waterhouse Coopers.(2007) .Carta Recordatoria. Impreso en Perú.

6. Vilchez Percy. (2004). Armonización de Normas Contables en un mundo global. Análisis y perspectivas. Trabajo Nacional para el Congreso Nacional de Contadores Públicos. Perú. 